\title{
Effect of Wettability Changes on Evaporation Rate and the Permeability Impairment due to Salt Deposition
}

\author{
Ayorinde Rufai ${ }^{1,2}$ and John Crawshaw ${ }^{1,2}$ \\ ${ }^{1}$ Department of Chemical Engineering, Imperial College London, London SW7 2AZ, United Kingdom; \\ ${ }^{2}$ Qatar Carbonates and Carbon Storage Research Centre, Imperial College London, London, UK. SW7 2AZ
}

Keywords: Brine, Drying, Wettability, Liquid films, Micromodel, Contact Angle

\begin{abstract}
Pore-scale visualization was employed to investigate evaporative drying of brine and associated salt deposition at different wetting conditions, using a $2.5 \mathrm{D}$ etchedsilicon/glass micromodel based on a thin section image of a carbonate rock. We also compared air drying with $\mathrm{CO}_{2}$ drying, with the latter having important applications in $\mathrm{CO}_{2}$ sequestration processes. The resulting permeability impairment was also measured.

For deionized-water in a water-wet model, we observed the three classical periods of evaporation: the constant rate period ( $C R P)$, the falling rate period (FRP) and the receding front period (RFP). The length of the deionized-water CRP was much shorter for a uniformly oil-wet model, but mixed wettability made little difference to the drying process. For brine systems at all wetting conditions, the dry area became linear with the square root of time after a short CRP. Although this is due to the deposited salt acting as a physical barrier to hydraulic connectivity, unlike the case of deionized-water which is due to capillary disconnection from the fracture channel.

For water-wet model, we observed two regions of a linear downward trend in the matrix and fracture permeability measurements. A similar trend was observed for the mixed-wet systems. However, for the oil-wet systems, fracture permeability only changes slightly even for $360 \mathrm{~g} / \mathrm{L}$ brine, a result of the absence of salt deposits in the fracture caused by the early rupture of the liquid wetting films needed to aid hydraulic connectivity. Overall, matrix permeability for all wetting conditions decreased with increasing brine concentration and was almost total for the $360 \mathrm{~g} / \mathrm{L}$ brine. Finally, using $\mathrm{CO}_{2}$ rather than air as carrier gas makes the brine phase more wetting especially in the deionized-water case, with the result that hydraulic connectivity was maintained for longer in the $\mathrm{CO}_{2}$ case compared to dry-out with air.
\end{abstract}




\section{Introduction}

Studies on evaporative drying from brine-saturated porous media are essential for many engineering and science processes, as it is important for applications such as soil and ground-water salinization, and in the preservation of building (masonry) materials. It is also important in geological applications such as enhanced oil recovery and $\mathrm{CO}_{2}$ sequestration in underground reservoirs. When under-saturated gas passes across the face of a porous medium, as is the case for exposed soil, building materials or $\mathrm{CO}_{2}$ injection into fractured formations, three stages of evaporation are often observed. An early stage of evaporation characterized by a relatively high and constant evaporation rate, known as stage-1 evaporation or the Constant Rate Period (CRP), is followed by a much lower evaporation rate known as the Falling Rate Period (FRP), and the receding front period (RFP) ${ }^{1-4}$. The claim that the CRP is negligible even for deionized water drying was made over thirty-five years ago ${ }^{3}$. This claim has however been disproved by many studies including this present study ${ }^{1,3-12}$. The falling rate period and the receding rate period are often combined together as stage-2 evaporation ${ }^{6,13-15}$.

This behaviour has been extensively analysed for the case of porous media saturated with pure water. Evaporation rate is high during the CRP due to the high initial water content. Many rocks and untreated, porous building materials are preferentially water-wet and capillary forces transport water via corner wetting films to the evaporating surface during this first stage of drying. Hence, the CRP is said to be controlled by external demand ${ }^{1}$. During the CRP, the porous media behaves as though it is virtually saturated because the capillary forces are strong enough to balance evaporative demand at the surface ${ }^{2}$. Eventually, gravity and viscous forces overcome the capillary driving force at some point, cutting hydraulic connectivity to the surface ${ }^{4}$, this marks the end of the CRP and the beginning of the FRP. The corner wetting menisci no longer reach the surface of the porous media and the drying front moves back from the surface to within the porous media. The FRP is characterized by a sharp drop in evaporation rate and limited by diffusion through the porous media. This quickly transitions from an advection-dominated period to a diffusion dominated period (RFP) with a further and prolonged drop in evaporation rate ${ }^{1-7,12-14,16}$. 
Drying of natural and artificial brine-containing porous media (saline aquifers, oil and gas reservoirs, etc. and porous building materials respectively) leads to the precipitation of the dissolved salt. Solid salts are deposited as crystals after evaporation of water from brine leading to super-saturation. Salt crystallization can occur outside (efflorescence) or within (sub-florescence) the porous media when the salt's solubility limit is reached. The main difference between these two phenomena is that primary efflorescence happens as a limiting case of the crystallization problem within pores ${ }^{17}$. The position of precipitated salts is dependent on the balance between advection towards the surface of the porous medium, driven by capillary forces, and diffusion of the salt away from high concentration regions, characterized by the Peclet number ${ }^{18-20}$. Efflorescence and sub-florescence and the problems that arise from it are the subjects of active research within the cement and concrete research communities ${ }^{1721-30}$.

Different phenomena affect the dynamics of the precipitated salt in a porous medium ${ }^{31-33}$. The effect of brine mobility and the initial liquid saturation has been studied ${ }^{34-37}$, showing that the drying fronts move with minute salt deposition for low brine concentration and low mobility. However, for high mobility brine, the drying font is continuously supplied with brine as a result of capillary pressure gradient leading to more salt precipitation ${ }^{34-37}$. The nature (crusty or patchy) ${ }^{38-42}$, position and distribution (efflorescence or sub-florescence) ${ }^{37,42-45}$ of the precipitated salts have been studied. Other factors affecting evaporation in porous media such as the effects of grain size, grain angularity, pore size and heterogeneity of porous media ${ }^{39}$, 41, 46-48 have also been studied. As well as the effect of confinement and system dimensions ${ }^{11,28,40,49-57}$, the effect of liquid film induced capillarity ${ }^{5,7,17,58-60}$ and the effect of the porous media wetting conditions ${ }^{8,10,42,52,61-62}$.

The precipitation of these salts can lead to disastrous damage in building and statutes made with porous materials ${ }^{21-22,24,26,29-30,56}$. This damage can be made worse by treatments that aim to waterproof the building materials as this can encourage sub-florescence that weakens the outer layer ${ }^{54}$. Pore-network models have also been used to study and predict drying of pure liquid porous media without salt precipitation ${ }^{58,63-66}$ and to study combined evaporation and salt precipitation in porous media ${ }^{28,67-68}$. Also, for $\mathrm{CO}_{2}$ sequestration processes, precipitation of salts, 
mainly consisting of halite $(\mathrm{NaCl})$, can be a serious source of problem during $\mathrm{CO}_{2}$ injection because this can lead to reductions in porosity and permeability of the reservoir in the vicinity of the wellbore ${ }^{19,34,69-74}$. Oil reservoirs have a wide range of wetting states ${ }^{75-77}$ and, given the strong influence of capillary forces outlined above, this may have a significant impact on halite deposition. The effect of these different porous media wetting states on the different stages of evaporation and the subsequently deposited salt is not well understood and remains one of the open problems in the field, especially during the storage of supercritical $\mathrm{CO}_{2}$ in depleted oil and gas reservoirs. Likewise, to the best of our knowledge, the influence of the composition of the gas carrying water vapour during drying in a salt contaminated porous media, for example drying with air versus drying with $\mathrm{CO}_{2}$ (as is the case during $\mathrm{CO}_{2}$ sequestration), has not been studied before. Understanding the influence of these factors on the pore-scale is essential in order to assess and optimize our carbon sequestration efforts.

Finally, due to the very intricate and complex nature of real porous media, simple artificial patterns can be used to understand the underlying phenomena occurring in them. Studies based on these simple models, such as the capillary bundle model ${ }^{78}$, have been shown to be very useful and they often act as a vital link to understanding the transport phenomena in real porous media $1,7,50,79-80$. In this study, we have increased the complexity of the artificial porous media beyond bundles of capillaries by working with micromodels that are based on a thin section of a sucrosic dolomite carbonate rock ${ }^{12,81}$. The use of micromodels also afforded us direct visual observation and imaging using imaging and optical spectroscopy in real time ${ }^{82}$. Hence, we have employed pore scale visualization to investigate evaporative drying and the associated salt deposition of $\mathrm{NaCl}$ brine in porous media at different atmospheric and wetting conditions, using a $2.5 \mathrm{D}$ etched-silicon/glass micromodel. A new technique for altering wettability will be presented as well as the reduction of permeability due to salt deposition. 


\section{Experimental Methods}

\section{Micromodels and Imaging Techniques}

The micromodels used in this study are the same as those used in a previous study 12 and are based on thin-section images of a sucrosic dolomite rock as an example of a simple carbonate structure. The pattern designs were derived from existing rock section images as pioneered by Hornbrook ${ }^{83}$ and Rangel-German \& Kovscek ${ }^{84}$. SEM images of some sections of the etched micromodel wafer are shown in figure 1.

(a)
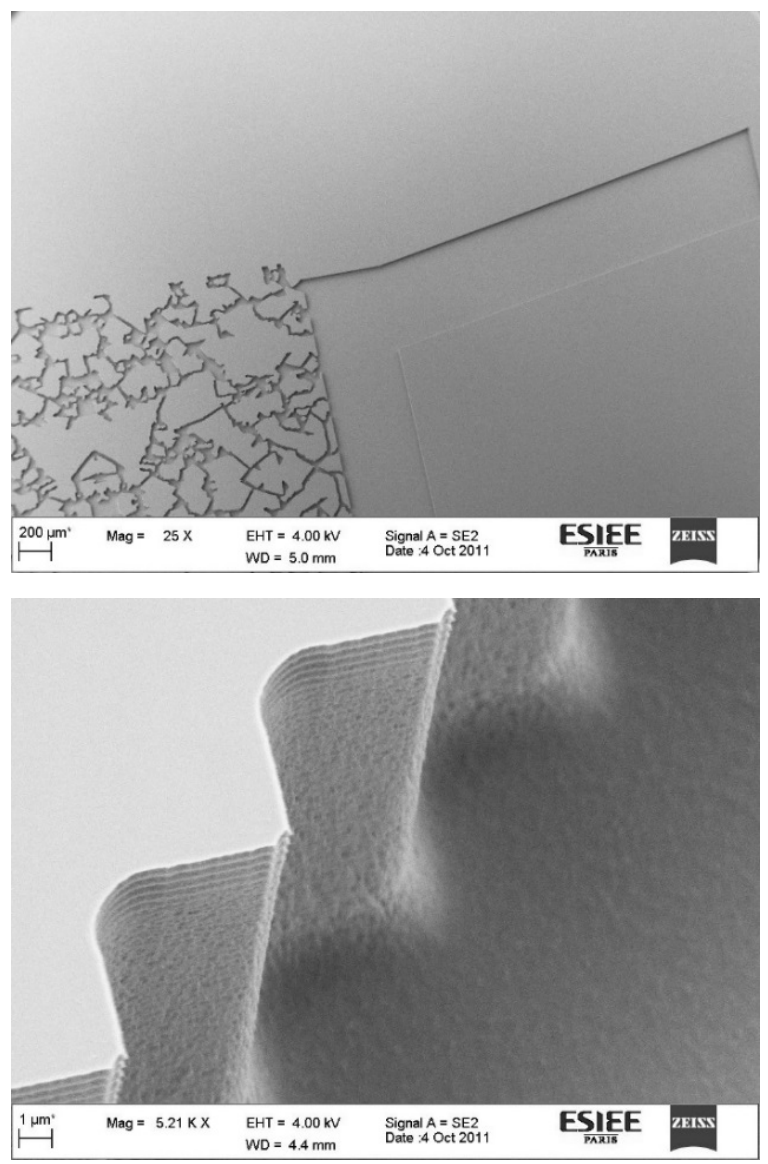

(b)

Figure 1. SEM images showing different sections of the sucrosic dolomite micromodel wafer at different magnification. (a) Section of the mask showing the inlet/outlet channel, the fracture channel and some matrix. (b) Magnified SEM image of the mask showing the depth of the micromodel. 
The micromodel consist of two fluid distribution channels (on both ends of the matrix) and four ports located in each corner of the flow channel for fluid injection and production. The micromodel matrix size is $2.5 \times 1 \mathrm{~cm}^{2}$ etched area with constant etch depth of $25 \mu \mathrm{m}$. The duct width at each end is $500 \mu \mathrm{m}$ and the matrix grain size ranges between $6-1250 \mu \mathrm{m}$. The average porosity measured from the binarised model image was estimated to be 0.28 and total pore area (excluding vertical channels) is approximately $0.7 \mathrm{~cm}^{2}$. The coordination number varied between 1 and 4 , with matrix permeability of about $350 \mathrm{mD}^{12}$. The pore-body and throat size distributions, estimated from the equivalent circular diameter and distance across the throats respectively, are shown in the plot in figure 2. We employed optical imaging to obtain the images reported in this study. A DSLR (digital single-lens reflex) camera (D800E, Nikon) combined with a macro-lens (AF-S Micro Nikkor 105 mm, Nikon) and filter (Pro1 Digital Close-Up No.3, Hoya) was used to capture images that require a wide field of view and rapid image acquisition. High-resolution images were obtained using an inverted light microscope (AxioObserver A1.m, Zeiss). More explicit details of the micromodel and the imaging techniques are provided in a recently published paper ${ }^{12}$.

(a)

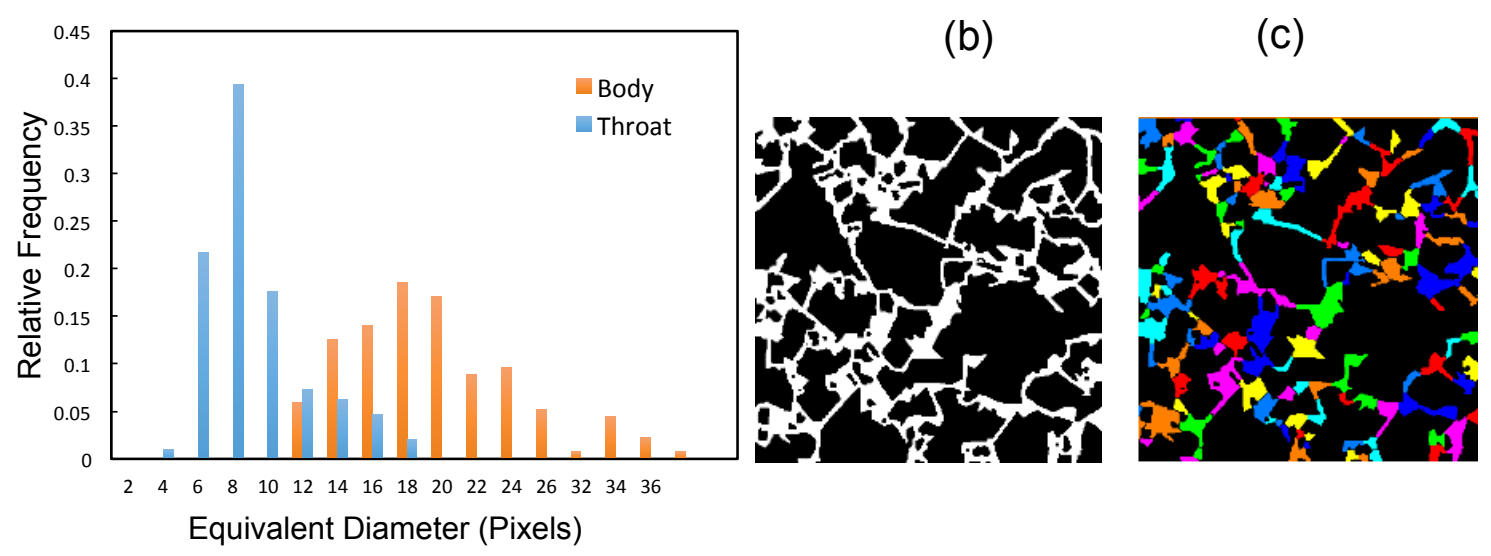

Figure 2. (a) Micromodel repeat unit pore-body and throat size distributions in pixels (2.5um/pixel), (b) Binary image of the thin section of the sucrosic dolomite mirrored and repeated throughout the micromodel $(0.16 \mathrm{X}$ $0.16 \mathrm{~cm}$ ), (c) colour-coded image of the repeat unit showing the separation of the etched region into individual pores each of which is assigned a random colour.

\section{Micromodel Wettability Alteration Technique}


The micromodels are manufactured to be water-wetting. In order to produce mixedwet micromodels for drying experiments, an alternative technique for wettability alterations of micromodel was developed. Well-established wettability alteration techniques ${ }^{85-86}$ that rely on silane chemistry cannot be used to produce mixed-wet conditions as the silane reacts with the water phase when the model is saturated with both oil and water to generate the appropriate spatial distribution of wetting alteration. Alteration directly with crude oil ${ }^{75,87}$ was also tried, but this rendered the model opaque to visible light. The new technique involves injecting a solution containing silicone caulk (151 Products LTD) $(10 \mathrm{v} / \mathrm{v} \%)$ dissolved in Dodecane $(\geq$ $99 \%$, Sigma-Aldrich) (90 v/v \%) into a micromodel initially saturated with water. This ensures that only the surfaces coming into contact with the hydrocarbon phase have wettability alteration, as is the case in oil reservoirs ${ }^{75,87}$. The micromodel was left to age for 2 days at ambient conditions. After the treatment stage, the micromodel was then completely dried by passing dry compressed air through it after which it was placed in an oven at $60^{\circ} \mathrm{C}$ for 24 hours. A uniformly oil-wet model was also prepared by injecting the silicone solution into a dry model.

After altering the wettability of the micromodel as described in the experimental procedure, the validity of the technique was tested by measuring the contact angle of brine on glass in air using a goniometer (figure 3 ).

(a)

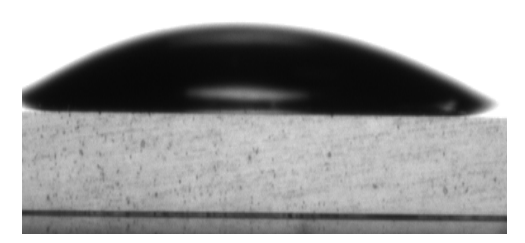

Figure 3. Saturated brine $(36 \mathrm{wt} \% \mathrm{NaCl})$ on glass slide before treatment (Contact angle: $\left.\approx 25^{\circ}\right)$ and after treatment (Contact angle: $\approx 108^{\circ}$ ).

(b)

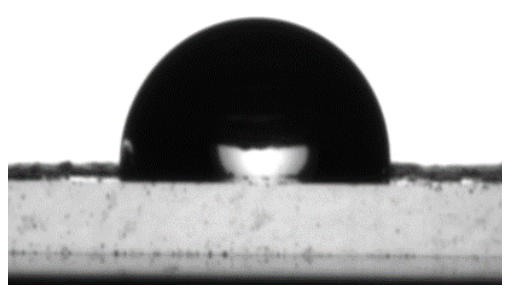

(a)
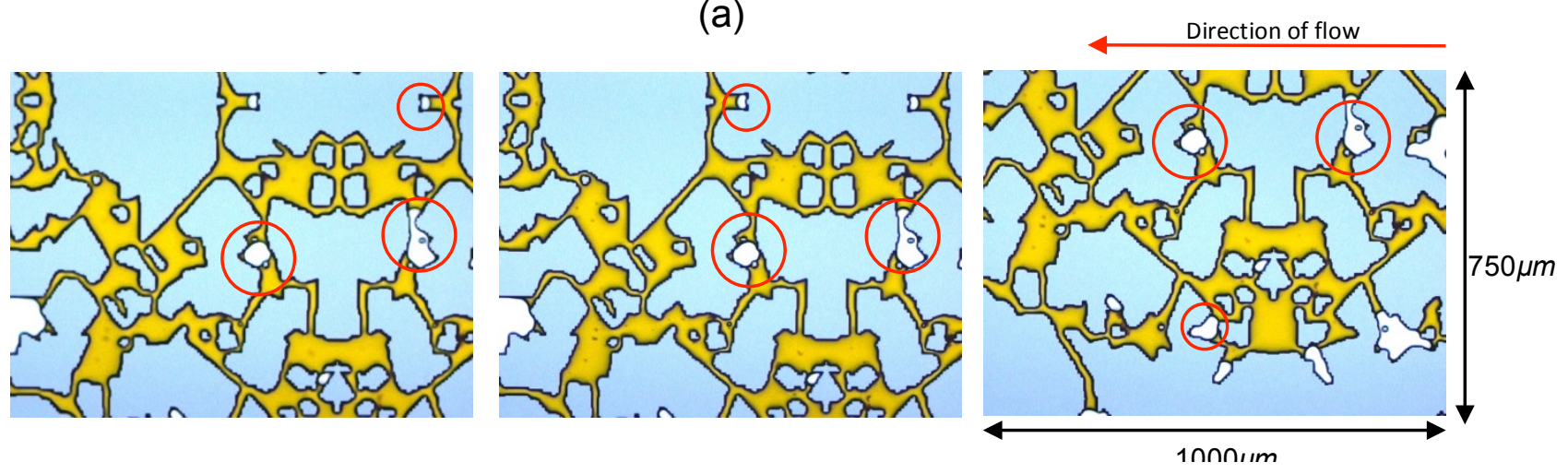


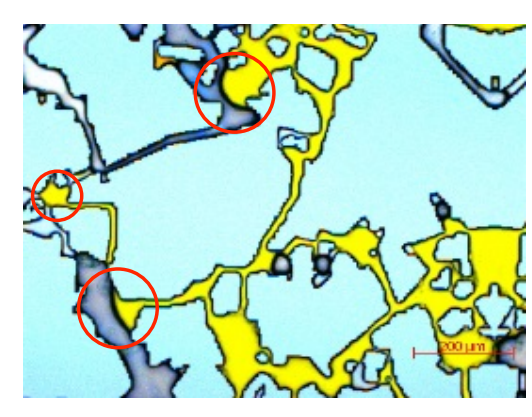

(b)

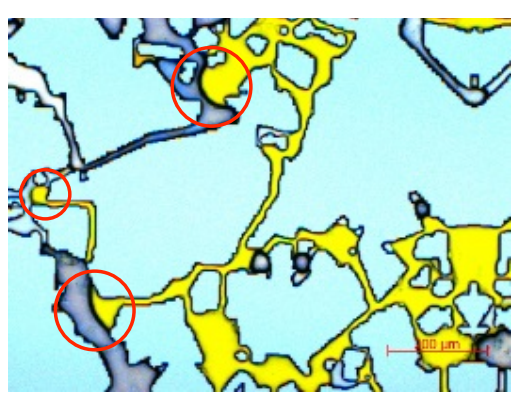

Direction of flow

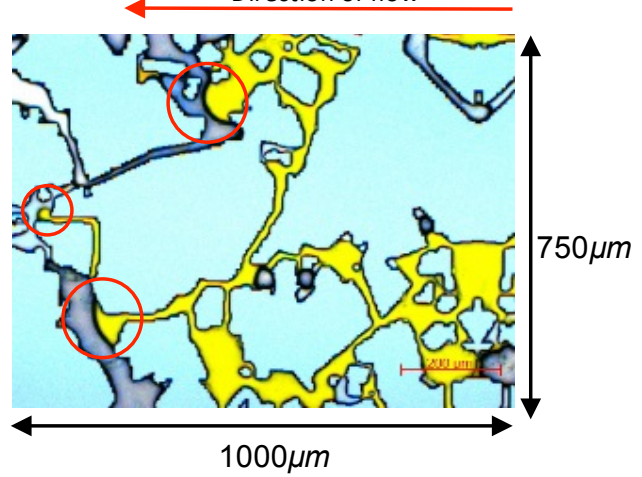

Figure 4. (a). Proof of model hydrophilicity before treatment, water invading fonts are concave (invading fonts are highlighted with red rings in the images), (b). Proof of model hydrophobicity, water invading fonts are convex (invading fonts are highlighted with red rings in the images).

The validity of the technique was also tested on the micromodel itself. The meniscus of the invading deionized-water (a very low concentration of fluorescein was added to the deionized-water for improved visualisation and easier phase differentiation) in the images in figure $4 \mathrm{~b}$ can be seen to be convex during forced imbibition confirming that the micromodel is no longer water-wet, unlike figure $4 a$ which shows the invading deionized-water to be concave in a water-wet micromodel.

\section{Experimental Procedure and Image Processing}

$\mathrm{NaCl}$ brine solutions were prepared freshly in the laboratory at each of the concentrations studied. Fluorescein (46960-25G-F, Sigma-Aldrich. 1:700,000 v/v) was added to each of the $\mathrm{NaCl}$ brine solutions for easier phase segmentation. The micromodel was affixed in a holder designed and fabricated in-house. Brine injection into micromodel was by a $10 \mathrm{ml}$ syringe (BD Plasipak, BD300013) with a programmable syringe pump (BS-80000, Braintree Scientific Ltd). To prevent the presence of trapped air bubbles, $\mathrm{CO}_{2}$ gas was first used to flush the air out of the micromodel before injecting the brine solutions.

Subsequently, many pore volumes of the brine solution were continuously injected to flush out the all the $\mathrm{CO}_{2}$ and the dissolved $\mathrm{CO}_{2}$-brine system in the micromodel 
before closing the valves to saturate the micromodel with only brine. This is to ensure that the $\mathrm{NaCl}$ brine solution used for drying is essentially free of any dissolved $\mathrm{CO}_{2}$. After achieving $100 \%$ brine saturation of the micromodel (checked with the high-resolution Inverted Light Microscope), the micromodel was placed horizontally (eliminating gravity effects) under the DSLR camera. The DSLR camera is placed on an adjustable stand facing vertically downwards and adjusted to capture the entire micromodel dimension in one image.

The dry gas line (air or $\mathrm{CO}_{2}$ ) was attached to one of the inlet ports at the side of the matrix selected for drying while its adjacent port was opened to the atmosphere for continuous flow of dry gas. To ensure evaporative drying, the dry gas pressure was set at 0.5 bars, resulting in a low enough flow rate. This was done to maintain very low relative humidity throughout the fracture channel and also circumvent forced advection into the matrix. The evolution of the dry-out was then captured at different time intervals and the movement of the drying front was observed. The images captured were saved in the TIFF format without compression, to obtain some quantitative data from the images, ImageJ was used to calculate the dry pore area (equivalent to mass lost by evaporation) of each of the captured images.

Since the brine was dyed with fluorescein, the dry pore areas are easily distinguishable from the pores still full of brine. Images were cropped and registered before segmentation, then the images were spatially calibrated using the known size of the micromodel pattern before setting the parameters to measure, in this case, "area". Colour-based thresholding was then used to segment the dry pore areas followed by its area measurement. The procedure for estimating the dry pore area from the images using ImageJ and some examples of this was highlighted in our recent paper $^{12}$. The quantitative data presented in this study are subject to uncertainty in dry pore area measurements. The average error was estimated by conducting repeat experiments and computing the standard deviation of the dry pore area measurements to be approximately $0.02 \mathrm{~cm}^{2}$. 


\section{Results and Discussion}

\section{Evaporative Drying of Saturated NaCl brine in a Water-wet Model}

The area of the matrix devoid of brine (dry pore area) was measured with the technique laid out in the preceding section. This dry pore area is equivalent to mass lost by evaporation and is plotted as a function of time and the square root of time for $36 \mathrm{wt} \%$ Brine (saturated $\mathrm{NaCl}$ brine) in figure 5 and 6 respectively. The very short CRP is shown in figure 5 , this lasted for approximately 40 s for the saturated brine. Also, salt crystals start to deposit at the fracture channel along the outside edge of the porous matrix almost immediately. 


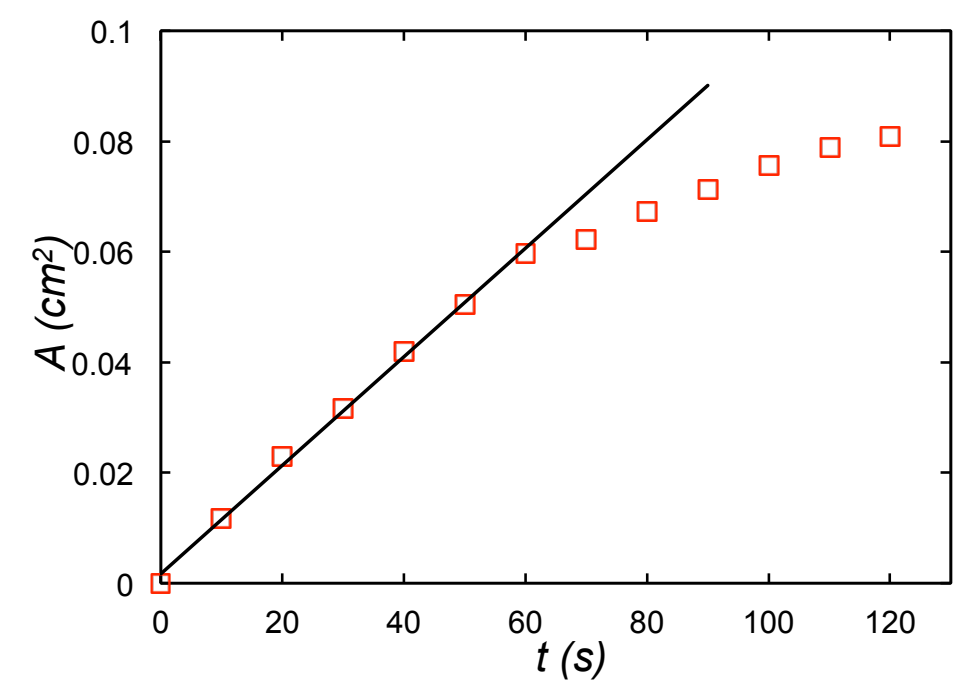

Figure 5. Early time plot of dry pore area as a function of time fitted with a linear function.

Drying of brine in a porous media is usually followed by the deposition of salt on the surface of the porous media (efflorescence) or within the porous media (subflorescence). Brine concentration increases as water is lost to the atmosphere, until the solubility limit is exceeded, resulting in solid salt deposition. Figure 6 shows that the evolution of the evaporative drying is linear in the square root of time at all times after the initial deposition of salt. This type of behaviour is usually attributed to a drying process governed by diffusion of vapour through a growing, completely dry region, as is always the case for deionized-water evaporation studies. However, salt crystals were found to continue to grow in the fracture channel considerably later after the transition to the slow evaporation, as can be seen in the insets in figure 6 . This implies that hydraulic connectivity to the fracture was maintained by corner wetting films, although these features were too small to be seen directly in the images. Also, this hydraulic connectivity is possible because deposited salt has been found to be both water-wetting and porous ${ }^{12,33}$. 


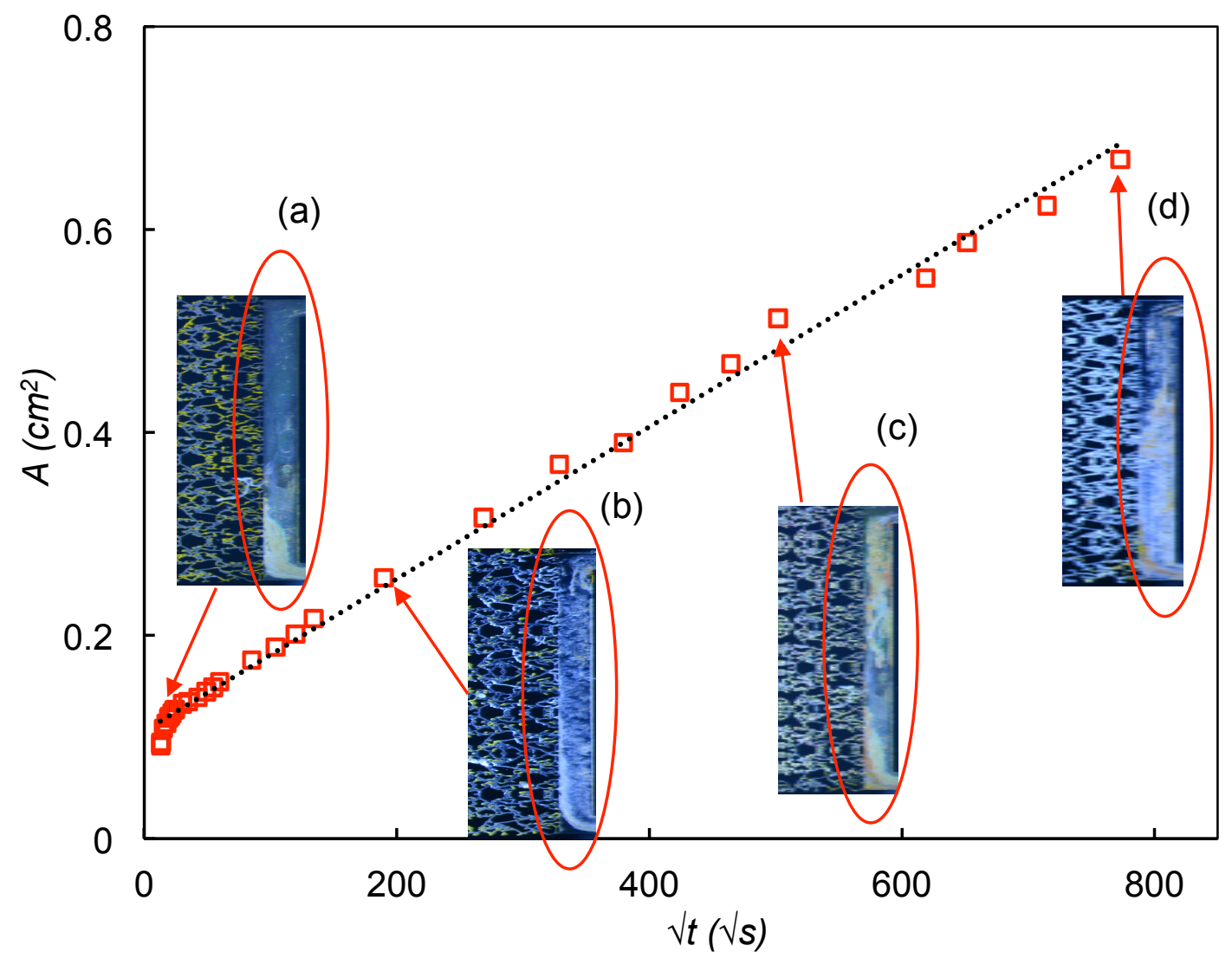

Figure 6. The plot of dry pore area as a function of $\sqrt{ } \mathrm{t}$ for $36 \mathrm{wt} \% \mathrm{NaCl}$ brine with insets showing precipitated salt crystals at the fracture at different times during drying. Images (a), (b), (c) and (d) with dimensions $\sim 0.12 \mathrm{~cm} x$ $1 \mathrm{~cm}$ were taken at approximately 60 seconds, 10hours, 3days and 7 days after the drying starts respectively. The red oval rings highlight the growth of salt (white crystals) in the fracture channel.

The relatively high viscosity of saturated $\mathrm{NaCl}$ brine (compared to deionized-water) provides a higher viscous resistance to mass transfer (liquid flow), and the development of dry solid salt patches in the fracture and matrix close to the fracture due to salt deposits plugging pores, are possible causes for the square root of time behaviour. The brine had a slightly larger contact angle on the silicon micromodel surfaces and this would result in the earlier detachment of the capillary connections to the outer surface. However, this cannot explain the behaviour while salt continues to deposit on the outside of the matrix.

\section{Effect of Porous Media Wettability on its Drying Dynamics}

The evaporative drying procedure used for the water-wet micromodel was repeated for the mixed-wet and oil-wet micromodel. Figure 7 shows the plot of normalised evaporation rate as a function of liquid saturation for the three wetting conditions at different salt concentrations. The classical periods of evaporation for porous media 
drying: the constant rate period (CRP), the falling rate period (FRP), and the receding front period (RFP) are clearly depicted in figure 7 . The evaporation rate $E$ is estimated from the change in dry pore area as a function of time while the liquid saturation $S$ is taken to be the area fraction of the pore space image occupied by brine. The key assumptions made in calculating the saturation are that pore spaces with liquid are filled and that dry pore areas are completely free of liquid. This allows the equivalence of the area fraction and saturation, which is a volumetric parameter.

All the plots follow a similar trend, a pseudo-constant high evaporation rate period, followed by a sharp fall in evaporation rate to a much slower evaporation rate period depicting the CRP, FRP and the RFP respectively. The depinning which signifies the CRP-FRP transition seems to happen a little early for the mixed-wet micromodel and much earlier for the oil-wet micromodel. Although, earlier experiments provide evidence that evaporation from mixed-wet porous media is slower than that from water-wet media ${ }^{8,10,16,42}$. The reason for this difference between the water-wet and mixed-wet micromodel is not apparent because we expect the matrix to have oil-wet patches where the micromodel surface came into contact with the oil phase during alteration, but still with water-wet corners. Hence, it is expected that the plots for both water-wet and mixed-wet model be very similar, especially if the water-wet corners form a continuous path (maintaining hydraulic connectivity through capillarity) to the evaporating surface.

We believe that the reason for the observed difference in the plots in early time as shown in figure $8 a$ is due to the presence of oil-wet patches at the evaporating surface. These oil-wet patches at the evaporating surface reduce the surface area available for evaporation at the surface, which in turn lowers the overall evaporation rate and increases overall drying time. Similarly, from studies of evaporation of $\mathrm{NaCl}$ solution from sand grains porous media of mixed wettability ${ }^{42}$, it was observed that the evaporative flux did not change considerably as the percentage of hydrophobic grains increases in the sand packs saturated with $\mathrm{NaCl}$ solution. It was concluded that the mixed wettability had little impact on the drying curves in sand packs ${ }^{42}$.

However, for the oil-wet micromodel, the constant rate period (CRP) was much shorter, even for deionized-water drying, due to the early depinning as seen in figure 7. This is expected since the oil-wet model cannot sustain capillarity at all as there 
are no corner wetting films as the contact angle was altered to greater than $90^{\circ}$. The CRP for the saturated brine in the oil-wet micromodel was almost non-existent, with a greatly prolonged FRP over a larger liquid saturation range.

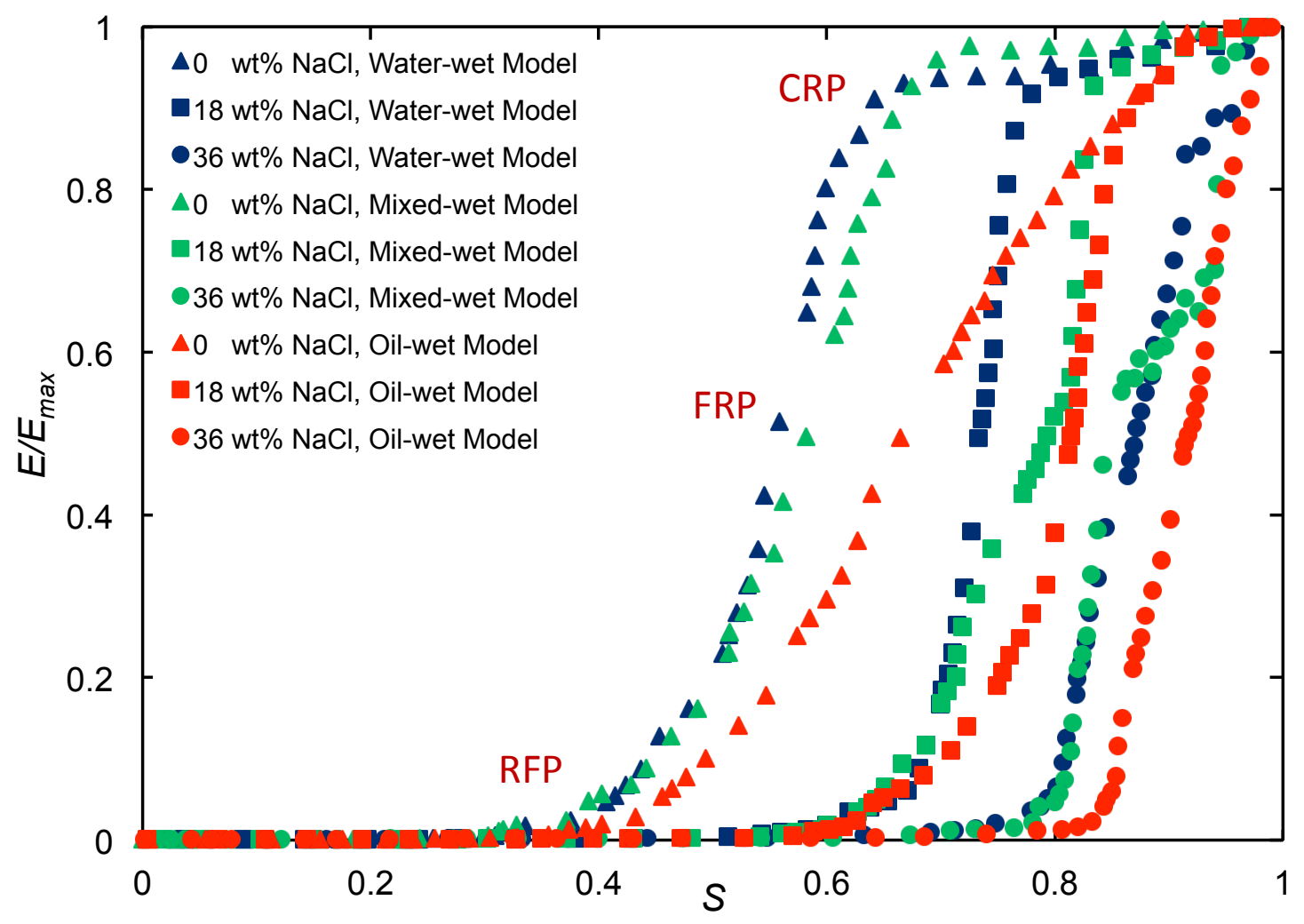

Figure 7. Evaporation rate as a function of liquid saturation for the three wetting states.

For all wetting conditions, in the experiments with brine, the dry area of the matrix became linear with the square root of time after the short CRP, as is shown in figure $8 \mathrm{~b}$. This behaviour is not due to capillary disconnection from the evaporating surface of the matrix as is usually the case for deionized-water drying (because the salt crystals continued to be deposited almost preferentially in the fracture above the matrix), but due to a combination of salt deposition in the fracture and in the matrix greatly impeding hydraulic connectivity to the evaporating surface as well as the high viscosity of the saturated brine increasing the viscous resistance to flow.

(a) 

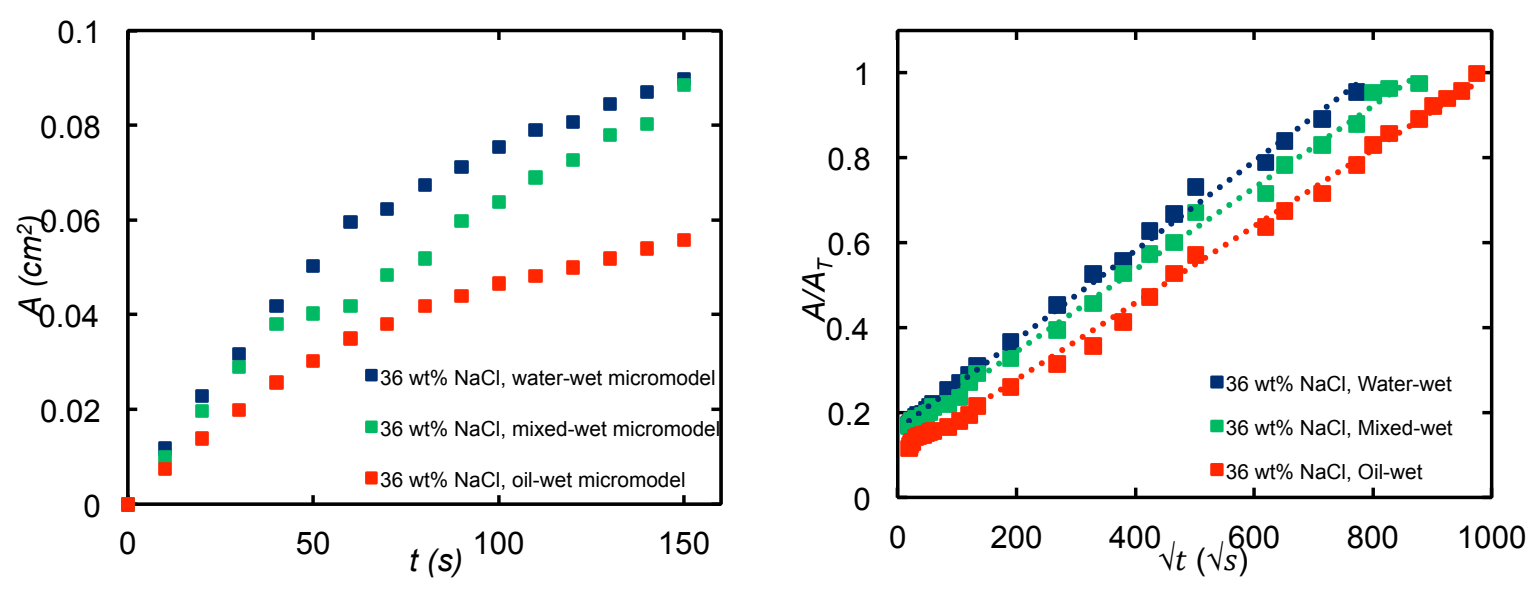

Figure 8. (a) Early-time dry area as a function of time and, (b). Dry area (normalised by total pore area) as a function of $\sqrt{t}$ for $36 \mathrm{wt} \% \mathrm{NaCl}$ brine for the different wetting conditions.

The effect of wettability on the precipitated salt distribution is more pronounced in the oil-wet micromodel. For the mixed-wet micromodel, the precipitated salt pattern was very similar to the water-wet micromodel case, with patchy salt formations in the fracture, again indicating that the corner menisci maintained hydraulic connectivity to the fracture well into the falling rate period. This was also confirmed by measuring the contact angle of saturated $\mathrm{NaCl}$ brine on a $\mathrm{NaCl}$ crystal using the goniometer. The crystal was completely wetting to saturated brine as the brine immediately spreads as soon as it touches the surface of the crystal, giving a contact angle of approximately $0^{\circ}$.

However, for the oil-wet micromodel, the hydraulic connectivity is broken very early into the drying and we have more patchy salt formations in the fracture as well as more salt precipitation in the matrix than was the case for the water-wet model. This is intuitive as there are no water-wet corners to maintain the capillary flow of liquid to the fracture. Therefore, the evaporation rate drops quickly and most of the salt is precipitated in the matrix. Overall, hydrophobicity in porous media leads to a reduction in capillary movement of water ${ }^{88}$.

\section{$\mathrm{CO}_{2}$ Dry-out Versus Air Dry-out}


To the best of our knowledge, the similarities and differences in the drying of porous media saturated with brine using air and $\mathrm{CO}_{2}$ have never been studied in the literature. The latter is particularly important in carbon sequestration process in saline aquifers.

The experimental procedure described above was repeated, but instead of injecting dry air, dry $\mathrm{CO}_{2}$ was injected across the fracture to effect evaporative drying of the brine in the micromodel. The outcome of the dry-out experiment with dry $\mathrm{CO}_{2}$ was qualitatively very similar to that of dry compressed air as can be seen in figure 9 . The trends of the different graphs plotted for the two systems were the same. However, there were some noticeable differences in the time it took to achieve complete drying. The $\mathrm{CO}_{2}$ system dried-out faster than the compressed air system. Also, the $\mathrm{CRP}$ for deionized-water was much longer for the dry $\mathrm{CO}_{2}$ system as seen in figure 9.

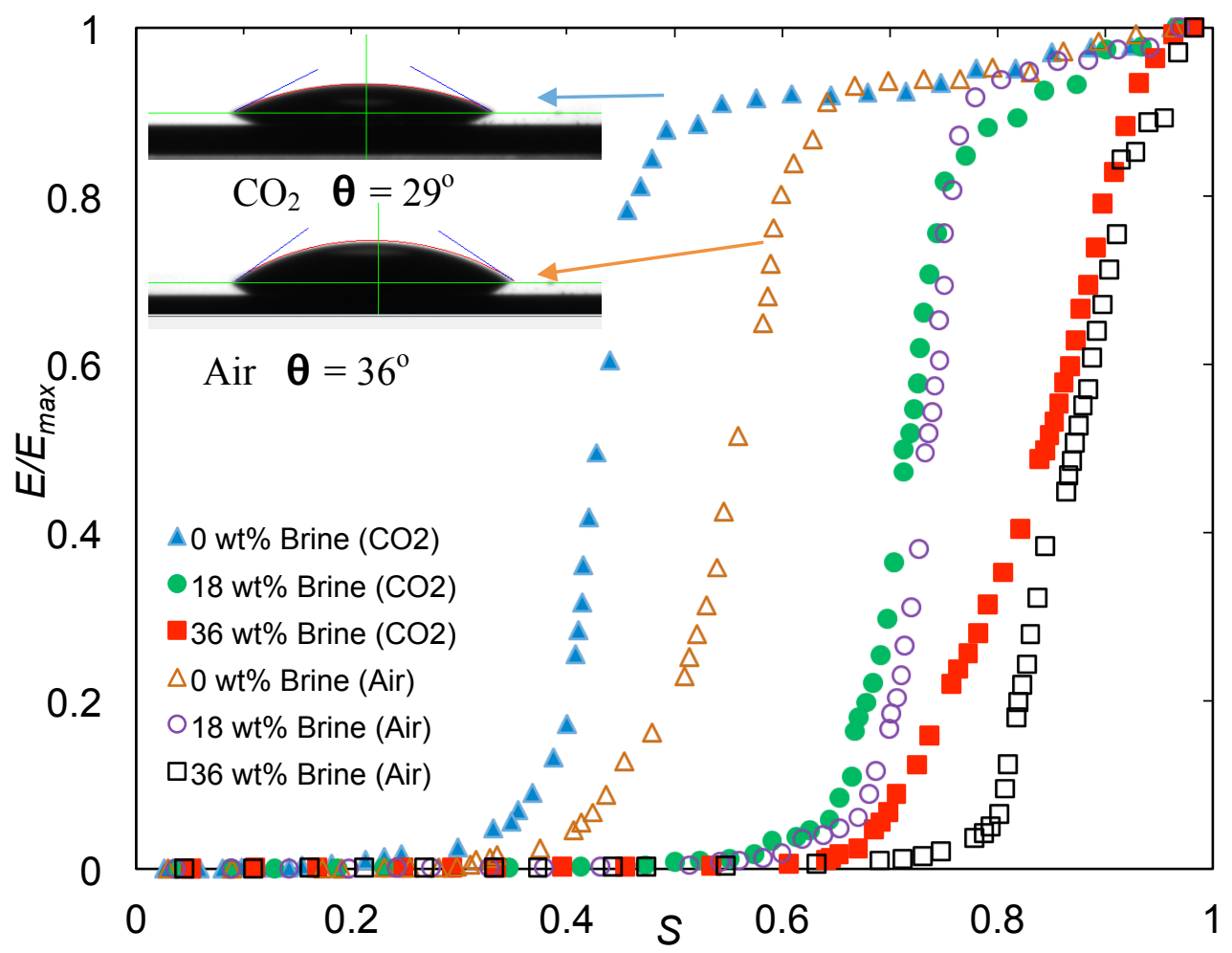

Figure 9. Evaporation rate as a function of liquid saturation for deionized-water, $18 \mathrm{wt} \%$ Brine, and $36 \mathrm{wt} \% \mathrm{NaCl}$ brine using dry air and $\mathrm{CO} 2$ as the drying medium. 
To further highlight this difference, the early time data for both systems were plotted on the same graph in figure 10. Clearly, the CRP for the $\mathrm{CO}_{2}$ systems was longer, although the difference is fairly small for the brines.

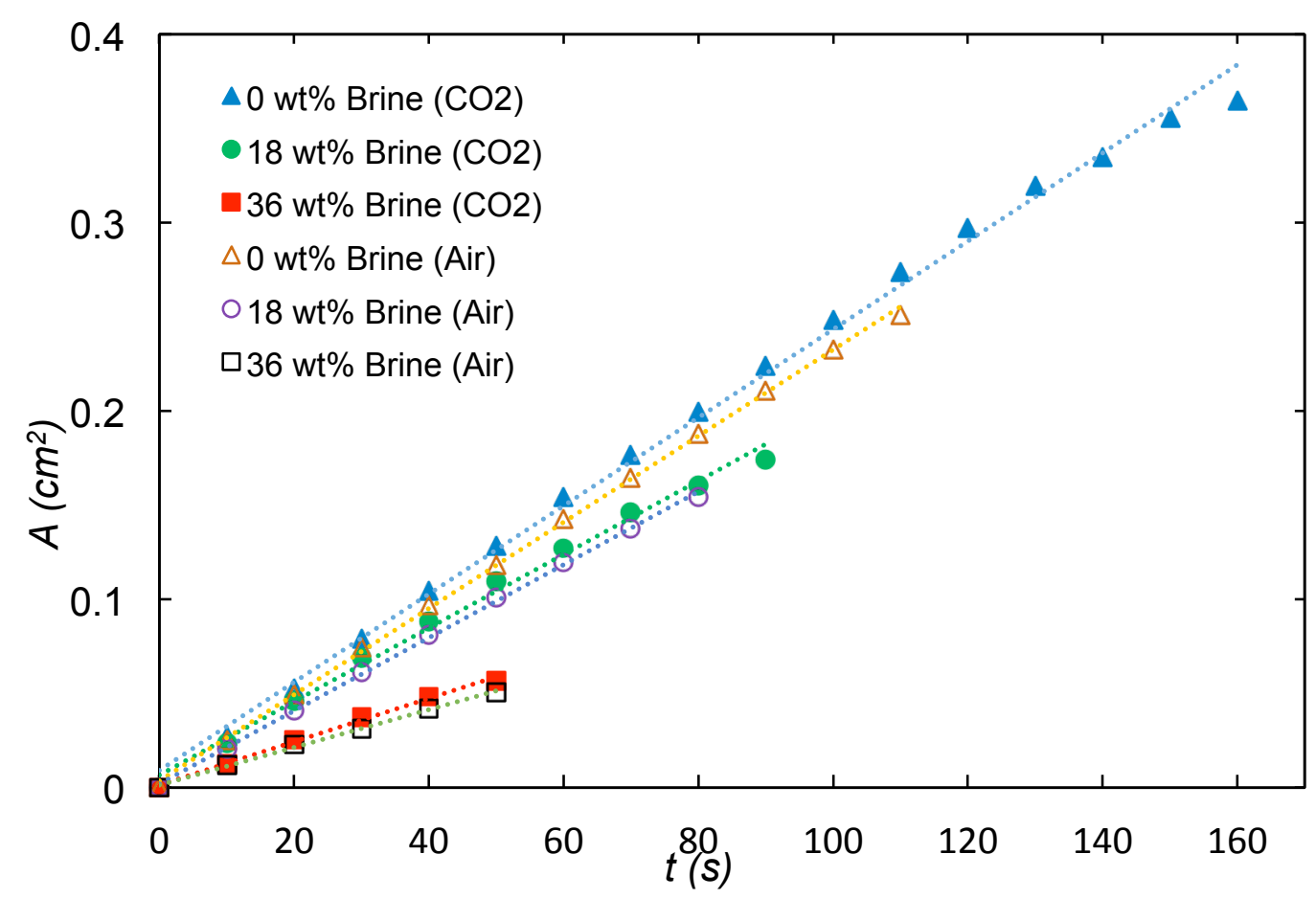

Figure 10. Comparison of CRPs for $\mathrm{CO} 2$ and Air dry-out, highlighting the difference as a consequence of changes in contact angle under the two systems.

The contact angle of deionized-water and saturated brine on glass and silicon were measured in air and $\mathrm{CO}_{2}$ with the result that the $\mathrm{CO}_{2}$ atmosphere makes the system more wetting, especially in the deionized-water case where the water contact angle measured in $\mathrm{CO}_{2}$ was $29^{\circ}$ compared to the contact angle of $34^{\circ}$ measured in air. This allows hydraulic connectivity to be maintained for slightly longer in the $\mathrm{CO}_{2}$ case and by extension, the Constant Rate Period (CRP) will be longer than was the case with compressed air. The $\mathrm{CO}_{2}$ atmosphere will make the corner films more effective in mass transfer of liquid to the evaporating surface, thereby decreasing the overall drying time ${ }^{9}$. The transition from the CRP for the saturated brine appears to depend less on wetting and more on the deposition of salt, so the small change in contact angle has less impact here. 


\section{Reduction in Permeability as a result of Salt deposition at Different Porous Media Wetting Conditions}

The injection of $\mathrm{CO}_{2}$ into saline aquifers results in the deposition of solid salt in the porous media. These solid salts clog and block the pore throats and pore bodies resulting in a reduction in porosity and by extension, a reduction in permeability of the aquifer. The salt deposition will happen mainly near the point of injection (injector wellhead) threatening the entire sequestration process, a process known as wellbore dry-out. This decrease in net injectivity can lead to increased injection costs, reduced carbon efficiency, and reduced $\mathrm{CO}_{2}$ storage potential ${ }^{73}$.

We studied the impairment of permeability caused by salt deposition at different porous media wetting conditions. The permeability changes were measured using two techniques. First, we considered fracture permeability by passing air through fracture (blue arrow in the image in figure 11a), while the ports at the opposite end of the micromodel were closed and the adjacent port open. In this configuration, air flowed in from one port, through the channel and out at the adjacent port, all on the same side of the micromodel. Secondly, we considered matrix permeability by injecting air into the matrix and opening the two ports at the opposite end of the micromodel, but closing the port adjacent to the air inlet port (see figure 11b). Air flow here was in from the inlet port, through the matrix and out at the opposite end ports (see blue arrow in figure 11b). These experimental procedures were carried out on micromodels that have been completely dried of different initial brine concentration at different wetting conditions. Permeability was computed by injecting air at different flow-rates and measuring the corresponding pressure difference using Darcy's Law.

(a)

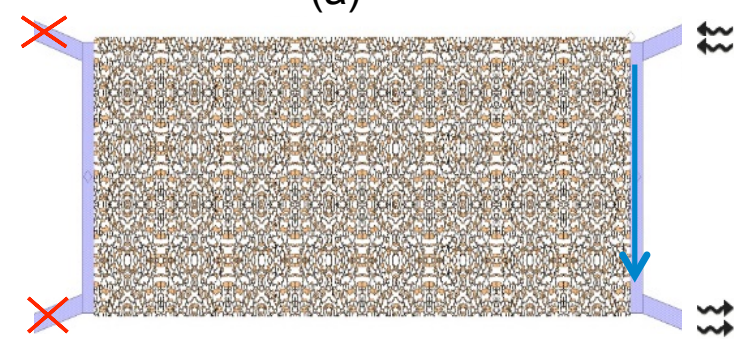

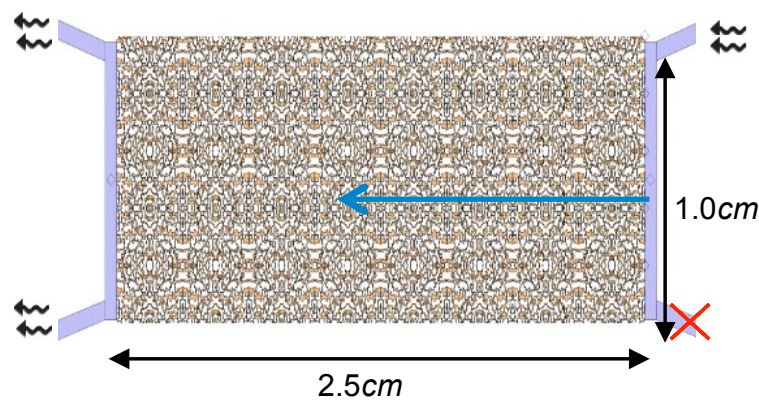

$2.5 \mathrm{~cm}$

Figure 11. Micromodel schematic showing the direction of air flow and the state of the valves at the inlet and outlet ports: (a) fracture permeability measurement (b) matrix permeability measurement. 
After measuring the permeability, we then defined permeability alteration as the ratio of final permeability to initial permeability ${ }^{19-20}$ and plotted it as a function of initial brine concentration (before drying) for fracture and matrix at different wetting conditions in figure 12. For the water-wet model, we observed two regions of a linear downward trend in the matrix and fracture permeability measurements first with the $120 \mathrm{~g} / \mathrm{L}$ brine to $180 \mathrm{~g} / \mathrm{L}$ brine before a sharp decrease and another downward trend from the $240 \mathrm{~g} / \mathrm{L}$ to the $360 \mathrm{~g} / \mathrm{L}$ brine. A similar trend was observed for the mixed-wet systems. However, for the oil-wet systems, the fracture permeability only changes slightly even for saturated brine $(360 \mathrm{~g} / \mathrm{L}$ brine). This is as a result of the absence of salt deposits in the fracture. The absence of salt deposits in the oil-wet is due to the lack of liquid wetting films needed to aid hydraulic connectivity to the fracture. Overall, the matrix permeability for all wetting conditions decreases with increasing brine concentration and is almost total for the saturated brine concentration $(360 \mathrm{~g} / \mathrm{L}$ brine). The practical consequence of this is that, in saline aquifers, salt deposition can lead to a significant decrease in injectivity.

(a)

Fracture Permeability

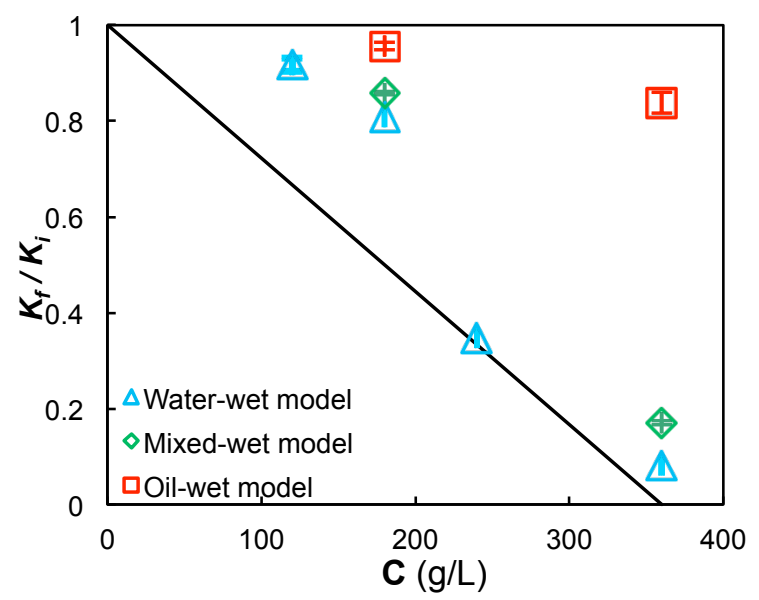

(b)

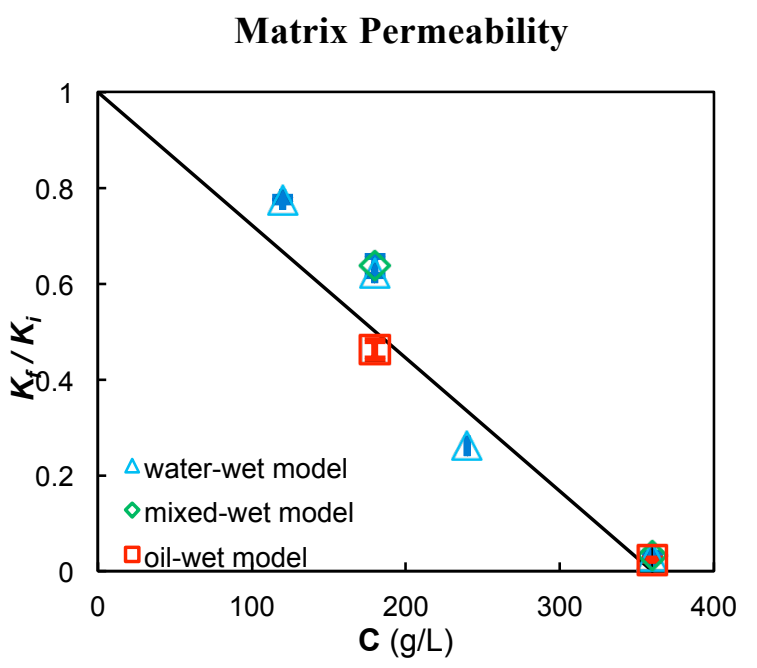

Figure 12. Permeability alteration versus initial salt concentration for the (a) fracture and, (b) matrix of the micromodel. 


\section{Conclusion}

Evaporation from water-wet micromodels based on the pore networks of natural rocks and saturated with $\mathrm{NaCl}$ brine was found to follow the well-established evolution of drying rate: an initially high, and almost constant, evaporation rate was followed by a transition to a much lower evaporation rate where the mass lost to evaporation becomes linear in the square-root of time. However, the transition into the low evaporation rate was not simply due to breaking the hydraulic connectivity to the evaporation surface (as is usually the case for deionized-water) as continuous deposition of salt was observed at the outer surface of the pore matrix well into the falling rate period.

The impact of the wetting state of the porous media on the drying dynamics was also studied. Two patterns of wettability alteration were obtained by treating the micromodel with a silicone caulk solution. A uniformly oil-wet state was produced by injecting the treatment into a dry micromodel and a mixed wet state, resembling the spatial distribution of wetting in an oil reservoir was produced by injecting the treatment into a model already saturated with water. The drying curve for water in the mixed-wet micro-model was very similar to that for the water-wet case, apart from the slightly earlier depinning in the mixed-wet condition. In the oil-wet micromodel, however, the evaporation rate dropped very soon after drying started, probably due to the early loss of hydraulic conductivity for this wetting state. The presence of oil-wet patches on the pore walls at the outer edge of the matrix would also reduce the evaporative flux by reducing the effective (water wetted) surface area available for evaporation and increase overall drying time.

The similarities and difference between air and $\mathrm{CO}_{2}$ as the drying agent for porous media drying were also studied. The drying curves are quite similar apart from the slightly later depinning in the case of $\mathrm{CO}_{2}$. This difference was investigated and concluded to be due to the $\mathrm{CO}_{2}$ system being slightly more water wet than the air system as evident in the contact angle of deionized-water under both conditions.

The matrix permeability, at complete dry-out for all wetting conditions, decreases with increasing salt concentration in a similar way. However, for the channel representing a fracture, the permeability decrease for the water-wet and mixed-wet cases was similar, while the fracture permeability for the oil-wet micromodel 
remained almost constant. This was a result of the absence of salt deposits in the fracture, caused by the lack of liquid wetting films needed to connect liquid brine to a point of evaporation in the fracture.

\section{Acknowledgments}

We gratefully acknowledge funding from the Petroleum Technology Development Fund (PTDF), provided by the Nigerian Government through the Presidential Special Nigeria Scholarship Scheme for Innovation and Development (PRESSID). We also gratefully acknowledge the equipment used for this research provided by the Qatar Carbonates and Carbon Storage Research Centre (QCCSRC), funded jointly by Qatar Petroleum, Shell, and Qatar Science and Technology Park. 


\section{References}

1. Chauvet, F.; Duru, P.; Geoffroy, S.; Prat, M., Three periods of drying of a single square capillary tube. Physical review letters 2009, 103 (12), 124502.

2. Scherer, G. W., Theory of drying. Journal of the American Ceramic Society 1990, 73 (1), 3-14.

3. Van Brakel, J., Mass transfer in convective drying. Advances in Drying 1980, 1, 217-267.

4. Lehmann, P.; Assouline, S.; Or, D., Characteristic lengths affecting evaporative drying of porous media. Physical Review E 2008, 77 (5), 056309.

5. Lehmann, P.; Or, D., Evaporation and capillary coupling across vertical textural contrasts in porous media. Physical Review E 2009, 80 (4), 046318.

6. Shokri, N.; Lehmann, P.; Or, D., Liquid-phase continuity and solute concentration dynamics during evaporation from porous media: Pore-scale processes near vaporization surface. Physical Review E 2010, 81 (4), 046308.

7. Chauvet, F.; Duru, P.; Prat, M., Depinning of evaporating liquid films in square capillary tubes: Influence of corners' roundedness. Physics of Fluids 2010, 22 (11), 112113.

8. Shahidzadeh-Bonn, N.; Azouni, A.; Coussot, P., Effect of wetting properties on the kinetics of drying of porous media. Journal of physics: condensed matter 2007, 19 (11), 112101.

9. Prat, M., On the influence of pore shape, contact angle and film flows on drying of capillary porous media. International journal of heat and mass transfer 2007, 50 (7), 1455-1468.

10. Chapuis, O.; Prat, M., Influence of wettability conditions on slow evaporation in twodimensional porous media. Physical review E 2007, 75 (4), 046311.

11. Prat, M.; Bouleux, F., Drying of capillary porous media with a stabilized front in two dimensions. Physical Review E 1999, 60 (5), 5647.

12. Rufai, A.; Crawshaw, J., Micromodel observations of evaporative drying and salt deposition in porous media. Physics of Fluids 2017, 29 (12), 126603.

13. Shokri, N.; Or, D., What determines drying rates at the onset of diffusion controlled stage-2 evaporation from porous media? Water Resources Research 2011, 47 (9).

14. Shokri, N.; Lehmann, P.; Or, D., Evaporation from layered porous media. Journal of Geophysical Research: Solid Earth 2010, 115 (B6).

15. Zuluaga, E.; Munoz, N.; Obando, G. In An experimental study to evaluate water vaporisation and formation damage caused by dry gas flow through porous media, International Symposium on Oilfield Scale, Society of Petroleum Engineers: 2001.

16. Shokri, N.; Lehmann, P.; Or, D., Characteristics of evaporation from partially wettable porous media. Water Resources Research 2009, 45 (2), W02415.

17. Le, D.; Hoang, H.; Mahadevan, J., Impact of capillary-driven liquid films on salt crystallization. Transport in porous media 2009, 80 (2), 229-252.

18. Azaroual, M.; Andre, L.; Peysson, Y.; Pironon, J.; Broseta, D.; Dedecker, F.; Egermann, P.; Desroches, J.; Hy-Billiot, J. In Behaviour of the $\mathrm{CO} 2$ injection well and the near wellbore during carbon dioxide injection in saline aquifers, TOUGH Symposium 2012, 2012; p Session V: Carbon Dioxide Storage II.

19. Peysson, Y., Permeability alteration induced by drying of brines in porous media. The European Physical Journal-Applied Physics 2012, 60 (2), 24206.

20. Peysson, Y.; Bazin, B.; Magnier, C.; Kohler, E.; Youssef, S., Permeability alteration due to salt precipitation driven by drying in the context of $\mathrm{CO} 2$ injection. Energy Procedia 2011, 4, 4387-4394.

21. Puyate, Y.; Lawrence, C.; Buenfeld, N.; McLoughlin, I., Chloride transport models for wick action in concrete at large Peclet number. Physics of fluids 1998, 10 (3), 566-575.

22. Scherer, G. W., Crystallization in pores. Cement and Concrete research 1999, 29 (8), 13471358.

23. Rodriguez-Navarro, C.; Doehne, E., Salt weathering: influence of evaporation rate, supersaturation and crystallization pattern. Earth surface processes and landforms 1999, 24 (3), 191209. 
24. Everett, D., The thermodynamics of frost damage to porous solids. Transactions of the Faraday society $1961,57,1541-1551$.

25. Goudie, A.; Viles, H. A., Salt weathering hazard. J. Wiley \& Sons: Chichester, 1997.

26. Scherer, G. W., Stress from crystallization of salt. Cement and concrete research 2004, 34 (9), 1613-1624.

27. Gupta, S.; Huinink, H. P.; Prat, M.; Pel, L.; Kopinga, K., Paradoxical drying of a fired-clay brick due to salt crystallization. Chemical Engineering Science 2014, 109, 204-211.

28. Huinink, H.; Pel, L.; Michels, M. v. A., How ions distribute in a drying porous medium: A simple model. Physics of fluids 2002, 14 (4), 1389-1395.

29. Steiger, M., Crystal growth in porous materials-l: the crystallization pressure of large crystals. Journal of crystal growth 2005, 282 (3), 455-469.

30. Shahidzadeh-Bonn, N.; Desarnaud, J.; Bertrand, F.; Chateau, X.; Bonn, D., Damage in porous media due to salt crystallization. Physical Review E 2010, 81 (6), 066110.

31. Rad, M. N.; Shokri, N.; Sahimi, M., Pore-scale dynamics of salt precipitation in drying porous media. Physical Review E 2013, 88 (3), 032404.

32. Shokri, N., Pore-scale dynamics of salt transport and distribution in drying porous media. Physics of Fluids 2014, 26 (1), 012106.

33. Shokri-Kuehni, S.; Vetter, T.; Webb, C.; Shokri, N., New insights into saline water evaporation from porous media: Complex interaction between evaporation rates, precipitation and surface temperature. Geophysical Research Letters 2017.

34. Bacci, G.; Korre, A.; Durucan, S., Experimental investigation into salt precipitation during $\mathrm{CO} 2$ injection in saline aquifers. Energy Procedia 2011, 4, 4450-4456.

35. Jambhekar, V.; Helmig, R.; Schröder, N.; Shokri, N., Free-flow-Porous-Media coupling for evaporation-driven transport and precipitation of salt in soil. Transport in Porous Media 2015, 110 (2), 251-280.

36. Hird, R.; Bolton, M., Measurement of Relative Humidity to Monitor Salt Migration in Unsaturated Porous Media. Transport in Porous Media 2016, 112 (3), 749-763.

37. Sghaier, N.; Prat, M.; Nasrallah, S. B., On ions transport during drying in a porous medium. Transport in porous media 2007, 67 (2), 243-274.

38. Shahidzadeh-Bonn, N.; Rafaï, S.; Bonn, D.; Wegdam, G., Salt crystallization during evaporation: impact of interfacial properties. Langmuir 2008, 24 (16), 8599-8605.

39. Veran-Tissoires, S.; Prat, M., Evaporation of a sodium chloride solution from a saturated porous medium with efflorescence formation. Journal of Fluid Mechanics 2014, 749, 701-749.

40. Veran-Tissoires, S.; Marcoux, M.; Prat, M., Discrete salt crystallization at the surface of a porous medium. Physical review letters 2012, 108 (5), 054502.

41. Hidri, F.; Sghaier, N.; Eloukabi, H.; Prat, M.; Nasrallah, S. B., Porous medium coffee ring effect and other factors affecting the first crystallisation time of sodium chloride at the surface of a drying porous medium. Physics of Fluids 2013, 25 (12), 127101.

42. Bergstad, M.; Shokri, N., Evaporation of $\mathrm{NaCl}$ solution from porous media with mixed wettability. Geophysical Research Letters 2016, 43 (9), 4426-4432.

43. Shokri, N.; Lehmann, P.; Or, D., Critical evaluation of enhancement factors for vapor transport through unsaturated porous media. Water resources research 2009, 45 (10), W10433.

44. Wong, V. N.; Dalal, R. C.; Greene, R. S., Salinity and sodicity effects on respiration and microbial biomass of soil. Biology and fertility of soils 2008, 44 (7), 943-953.

45. Norouzi Rad, M.; Shokri, N., Nonlinear effects of salt concentrations on evaporation from porous media. Geophysical Research Letters 2012, 39 (4), L04403.

46. Nachshon, U.; Weisbrod, N.; Dragila, M. I.; Grader, A., Combined evaporation and salt precipitation in homogeneous and heterogeneous porous media. Water Resources Research 2011, $47(3)$, w03513. 
47. Rad, M. N.; Shokri, N.; Keshmiri, A.; Withers, P. J., Effects of Grain and Pore Size on Salt Precipitation During Evaporation from Porous Media. Transport in Porous Media 2015, 110 (2), 281294.

48. Norouzi Rad, M.; Shokri, N., Effects of grain angularity on $\mathrm{NaCl}$ precipitation in porous media during evaporation. Water Resources Research 2014, 50 (11), 9020-9030.

49. Desarnaud, J.; Derluyn, H.; Molari, L.; de Miranda, S.; Cnudde, V.; Shahidzadeh, N., Drying of salt contaminated porous media: effect of primary and secondary nucleation. Journal of Applied Physics 2015, 118 (11), 114901.

50. Naillon, A.; Duru, P.; Marcoux, M.; Prat, M., Evaporation with sodium chloride crystallization in a capillary tube. Journal of Crystal Growth 2015, 422, 52-61.

51. Desarnaud, J.; Derluyn, H.; Carmeliet, J.; Bonn, D.; Shahidzadeh, N., Metastability limit for the nucleation of $\mathrm{NaCl}$ crystals in confinement. The journal of physical chemistry letters 2014, 5 (5), 890-895.

52. Eloukabi, H.; Sghaier, N.; Prat, M.; Ben Nassrallah, S., Drying experiments in a hydrophobic model porous medium in the presence of a dissolved salt. Chemical Engineering \& Technology 2011, 34 (7), 1085-1094.

53. Eloukabi, H.; Sghaier, N.; Nasrallah, S. B.; Prat, M., Experimental study of the effect of sodium chloride on drying of porous media: The crusty-patchy efflorescence transition. International Journal of Heat and Mass Transfer 2013, 56 (1), 80-93.

54. Sghaier, N.; Prat, M., Effect of efflorescence formation on drying kinetics of porous media. Transport in Porous Media 2009, 80 (3), 441-454.

55. Guglielmini, L.; Gontcharov, A.; Aldykiewicz Jr, A. J.; Stone, H. A., Drying of salt solutions in porous materials: intermediate-time dynamics and efflorescence. Physics of Fluids 2008, 20 (7), 077101.

56. DeCarlo, K. F.; Shokri, N., Salinity effects on cracking morphology and dynamics in 3-D desiccating clays. Water Resources Research 2014, 50 (4), 3052-3072.

57. Shokri, N.; Sahimi, M., Structure of drying fronts in three-dimensional porous media. Physical Review E 2012, 85 (6), 066312.

58. Yiotis, A.; Boudouvis, A.; Stubos, A.; Tsimpanogiannis, I.; Yortsos, Y., Effect of liquid films on the drying of porous media. AlChE Journal 2004, 50 (11), 2721-2737.

59. Ott, H.; Roels, S. M.; de Kloe, K., Salt precipitation due to supercritical gas injection: I. Capillary-driven flow in unimodal sandstone. International Journal of Greenhouse Gas Control 2015, 43, 247-255.

60. Ransohoff, T.; Radke, C., Laminar flow of a wetting liquid along the corners of a predominantly gas-occupied noncircular pore. Journal of colloid and interface science 1988, 121 (2), 392-401.

61. Shahidzadeh-Bonn, N.; Tournié, A.; Bichon, S.; Vié, P.; Rodts, S.; Faure, P.; Bertrand, F.; Azouni, A., Effect of wetting on the dynamics of drainage in porous media. Transport in porous media 2004, 56 (2), 209-224.

62. Bachmann, J.; Horton, R.; Van der Ploeg, R., Isothermal and nonisothermal evaporation from four sandy soils of different water repellency. Soil Science Society of America Journal 2001, 65 (6), 1599-1607.

63. Yiotis, A. G.; Tsimpanogiannis, I. N.; Stubos, A. K.; Yortsos, Y. C., Pore-network study of the characteristic periods in the drying of porous materials. Journal of Colloid and Interface Science 2006, 297 (2), 738-748.

64. Yiotis, A.; Stubos, A.; Boudouvis, A.; Tsimpanogiannis, I.; Yortsos, Y., Pore-network modeling of isothermal drying in porous media. Transport in Porous Media 2005, 58 (1), 63-86.

65. Yiotis, A. G.; Stubos, A.; Boudouvis, A.; Yortsos, Y. C., A 2-D pore-network model of the drying of single-component liquids in porous media. Advances in Water Resources 2001, 24 (3), 439-460.

66. Mahadevan, J.; Sharma, M. M.; Yortsos, Y. C., Flow-through drying of porous media. AlChE journal 2006, 52 (7), 2367-2380. 
67. Giorgis, T.; Carpita, M.; Battistelli, A., 2D modeling of salt precipitation during the injection of dry CO 2 in a depleted gas reservoir. Energy Conversion and Management 2007, 48 (6), 1816-1826.

68. Hurter, S.; Berge, J. G.; Labregere, D. In Simulations for $\mathrm{CO} 2$ injection projects with compositional simulator, Offshore Europe, Society of Petroleum Engineers: 2007.

69. Schutjens, P. M. T. M.; Hanssen, T. H.; Hettema, M. H. H.; Merour, J.; de Bree, P.; Coremans, J. W. A.; Helliesen, G., Compaction-Induced Porosity/Permeability Reduction in Sandstone Reservoirs: Data and Model for Elasticity-Dominated Deformation.

70. Pruess, K.; Müller, N., Formation dry-out from $\mathrm{CO} 2$ injection into saline aquifers: 1 . Effects of solids precipitation and their mitigation. Water Resources Research 2009, 45 (3), W03402.

71. Pruess, K.; Garcia, J., Multiphase flow dynamics during CO 2 disposal into saline aquifers. Environmental Geology 2002, 42 (2), 282-295.

72. Ott, H.; De Kloe, K.; Marcelis, F.; Makurat, A., Injection of supercritical $\mathrm{CO} 2$ in brine saturated sandstone: Pattern formation during salt precipitation. Energy Procedia 2011, 4, 4425-4432.

73. Kim, M.; Sell, A.; Sinton, D., Aquifer-on-a-Chip: understanding pore-scale salt precipitation dynamics during CO 2 sequestration. Lab on a Chip 2013, 13 (13), 2508-2518.

74. Muller, N.; Qi, R.; Mackie, E.; Pruess, K.; Blunt, M. J., CO2 injection impairment due to halite precipitation. Energy procedia 2009, 1 (1), 3507-3514.

75. Al-Menhali, A. S.; Krevor, S., Capillary Trapping of $\mathrm{CO} 2$ in Oil Reservoirs: Observations in a Mixed-Wet Carbonate Rock. Environmental Science \& Technology 2016, 50 (5), 2727-2734.

76. Austad, T.; Standnes, D. C., Spontaneous imbibition of water into oil-wet carbonates. Journal of Petroleum Science and Engineering 2003, 39 (3), 363-376.

77. Kasiri, N.; Bashiri, A., Wettability and Its Effects on Oil Recovery in Fractured and Conventional Reservoirs. Petroleum Science and Technology 2011, 29 (13), 1324-1333.

78. Marle, C., Multiphase flow in porous media. Éditions technip: 1981.

79. Squires, T. M.; Quake, S. R., Microfluidics: Fluid physics at the nanoliter scale. Reviews of modern physics 2005, 77 (3), 977.

80. Camassel, B.; Sghaier, N.; Prat, M.; Nasrallah, S. B., Evaporation in a capillary tube of square cross-section: application to ion transport. Chemical engineering science 2005, 60 (3), 815-826.

81. Knackstedt, M.; Arns, C.; Sheppard, A.; Senden, T.; Sok, R.; Cinar, Y.; Olafuyi, A.; Pinczewski, W. V.; Padhy, G.; loannidis, M., Pore scale analysis of electrical resistivity in complex core material. Proc. Of the Soc. Core Analysts, Calgary, Canada 2007.

82. Karadimitriou, N.; Hassanizadeh, S., A review of micromodels and their use in two-phase flow studies. Vadose Zone Journal 2012, 11 (3), 0072.

83. Hornbrook, J.; Castanier, L.; Pettit, P. In Observation of foam/oil interactions in a new, highresolution micromodel, SPE Annual Technical Conference and Exhibition, Society of Petroleum Engineers: 1991.

84. Rangel-German, E.; Kovscek, A., A micromodel investigation of two-phase matrix-fracture transfer mechanisms. Water resources research 2006, 42 (3), W03401.

85. Grate, J. W.; Warner, M. G.; Pittman, J. W.; Dehoff, K. J.; Wietsma, T. W.; Zhang, C.; Oostrom, M., Silane modification of glass and silica surfaces to obtain equally oil-wet surfaces in glass-covered silicon micromodel applications. Water Resources Research 2013, 49 (8), 4724-4729.

86. Esmaeilzadeh, P.; Sadeghi, M. T.; Fakhroueian, Z.; Bahramian, A.; Norouzbeigi, R., Wettability alteration of carbonate rocks from liquid-wetting to ultra gas-wetting using TiO 2, SiO 2 and CNT nanofluids containing fluorochemicals, for enhanced gas recovery. Journal of Natural Gas Science and Engineering 2015, 26, 1294-1305.

87. Alhammadi, A. M.; AlRatrout, A.; Singh, K.; Bijeljic, B.; Blunt, M. J., In situ characterization of mixed-wettability in a reservoir rock at subsurface conditions. Scientific Reports 2017, 7, 10753.

88. Sahimi, M., Flow and transport in porous media and fractured rock: from classical methods to modern approaches. John Wiley \& Sons: 2011. 